Supporting Information

\title{
Cesium Salts as Mild Chemical Scissors to Trim Carbon Nitride for Photocatalytic $\mathrm{H}_{2}$ Evolution
}

Wenming Xua $\mathrm{u}^{\mathrm{a}}$ Xianghui An ${ }^{\mathrm{a}}$, Qinggang Zhanga ${ }^{\mathrm{a}}$,Zhen $\mathrm{Li}^{\mathrm{a}}$, Qinhua Zhang ${ }^{\mathrm{a}}$, Zheng $\mathrm{Yao}^{\mathrm{a}}$, Xiaokai Wang ${ }^{\mathrm{a}}$, Sha Wang ${ }^{\mathrm{b}}$, Jingtang Zheng ${ }^{\mathrm{a}}$, Jing Zhang ${ }^{\mathrm{c}}$, Wenting $\mathrm{Wu}^{\mathrm{a} *}$ and Mingbo $\mathrm{Wu}^{\mathrm{a} *}$

${ }^{a}$ State Key Laboratory of Heavy Oil Processing, College of Chemical Engineering, China University of Petroleum (East China), No. 66 Changjiang West Road, Qingdao 266580, P. R. China

b Jiangsu Provincial Key Laboratory of Pulp and Paper Science and Technology, Nanjing Forestry University, No.159 Longpan Road, Nanjing 210037, China

${ }^{\mathrm{c}}$ State Key Laboratory of Safety and Control for Chemicals, SINOPEC Research Institute of Safety Engineering, No. 339 Songling Road, Qingdao, 266071, China

Corresponding email: wuwt@upc.edu.cn (Wenting Wu) wumb@upc.edu.cn (Mingbo Wu)

Number of Pages : 11

Number of Figures : 14

Number of Tables : 5 

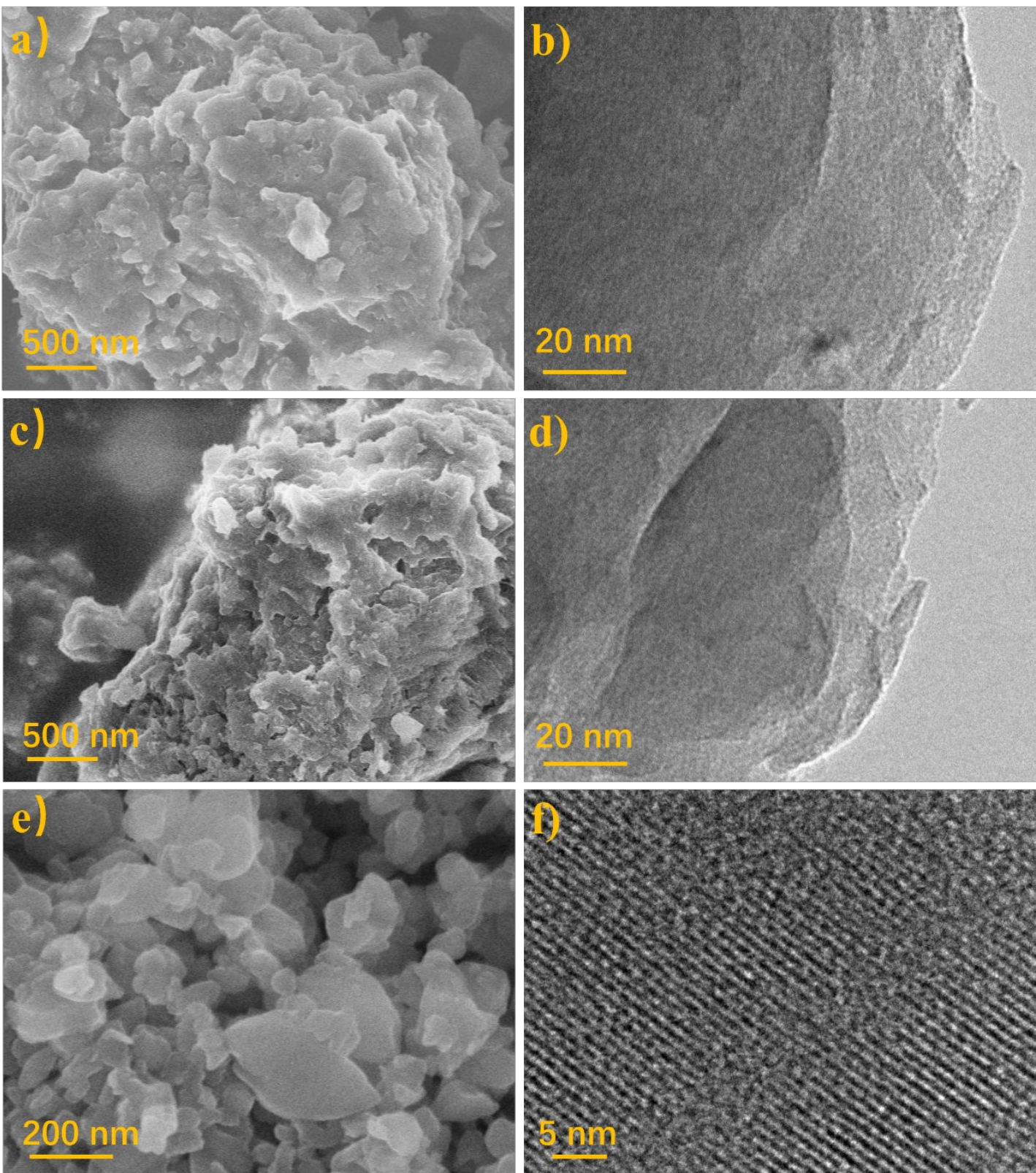

Figure S1. (a) SEM and (b) high-resolution TEM images of CN. (c) SEM and (d) highresolution TEM images of CN-2h. (e) SEM and (f) high-resolution TEM images of D-Cs-CN.

Table S1. The BET surface areas of samples

\begin{tabular}{ccccc}
\hline Samples & CN & CN-2h & Cs-CN-2h & D-Cs-CN \\
\hline BET surface areas $\left(\mathrm{m}^{2} \mathrm{~g}^{-1}\right)$ & 10.10 & 12.26 & 40.82 & 34.71 \\
\hline
\end{tabular}



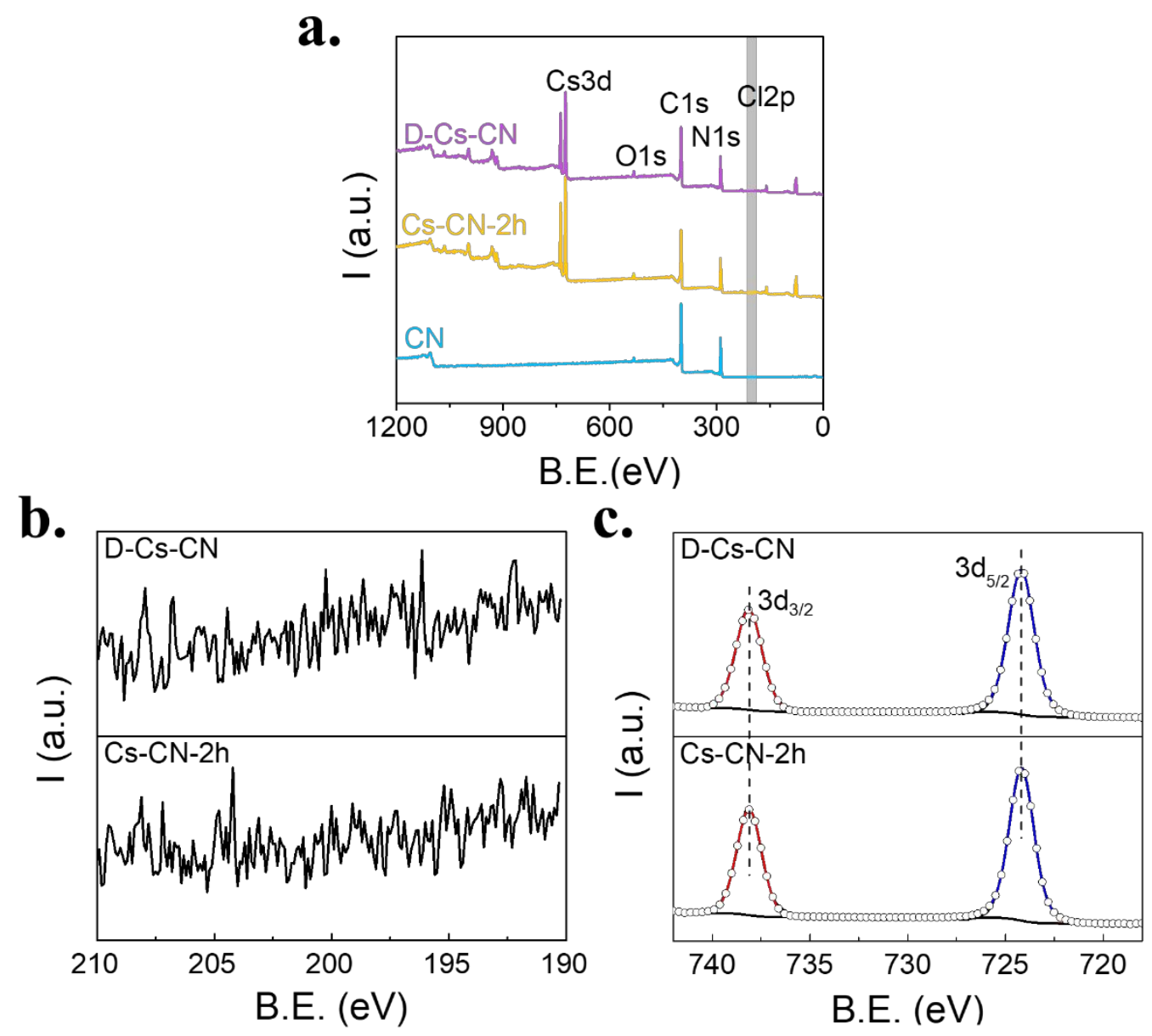

Figure S2. (a) The XPS of samples. The high resolution XPS Cl 2p (b) and Cs 3d (c) of Cs$\mathrm{CN}-2 \mathrm{~h}$ and D-Cs-CN.

Table S2. The peak analysis from high resolution XPS N 1s.

\begin{tabular}{ccccccc}
\hline \multirow{2}{*}{ Samples } & & $\mathrm{N} 1$ & $\mathrm{~N} 2$ & $\mathrm{~N} 3$ & Charge & N \\
& & $(\mathrm{C}-\mathrm{N}=\mathrm{C})$ & $\left(\mathrm{N}-\mathrm{C}_{3}\right)$ & $(\mathrm{C}-\mathrm{N}-\mathrm{H})$ & effect & \\
\hline \multirow{2}{*}{$\mathrm{CN}$} & Area & 58379.25 & 4898.33 & 2955.01 & 2457.93 & -- \\
& Percent (\%) & 84.99 & 7.13 & 4.30 & 3.58 & -- \\
\hline \multirow{2}{*}{ Cs-CN-2h } & Area & 45703.34 & 7992.19 & 3707.41 & 2682.27 & 1871.52 \\
& Percent (\%) & 73.77 & 12.90 & 5.98 & 4.33 & 3.02 \\
\hline \multirow{2}{*}{ D-Cs-CN } & Area & 35388.72 & 7005.48 & 5177.36 & 2913.68 & 2137.25 \\
& Percent (\%) & 72.48 & 11.19 & 8.27 & 4.65 & 3.41 \\
\hline
\end{tabular}


Table S3. The elements contents of samples by elemental analysis.

\begin{tabular}{ccccc}
\hline Samples & H (wt.\%) & C (wt.\%) & N (wt.\%) & n(C)/n(N) \\
\hline CN & 1.79 & 34.63 & 61.98 & 0.652 \\
CN-2h & 1.65 & 34.69 & 61.69 & 0.656 \\
Cs-CN-2h & 1.98 & 23.54 & 38.95 & 0.705 \\
D-Cs-CN & 1.90 & 22.87 & 38.09 & 0.700 \\
\hline
\end{tabular}

Table S4. The elemental composition from XPS.

\begin{tabular}{cccccc}
\hline Samples & C atm.\% & N atm.\% & O atm.\% & Cs atm.\% & Cl atm.\% \\
\hline CN & 43.27 & 54.36 & 2.37 & -- & -- \\
CN-2h & 44.48 & 53.09 & 2.43 & -- & -- \\
Cs-CN-2h & 42.29 & 48.33 & 4.12 & 4.80 & 0.27 \\
D-Cs-CN & 43.81 & 48.27 & 3.46 & 4.11 & 0.25 \\
\hline
\end{tabular}

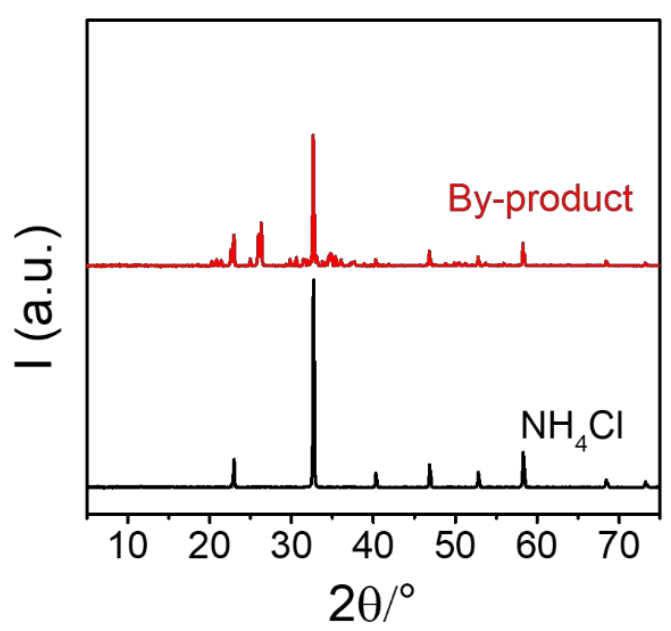

Figure S3. XRD patterns of by-product of Cs-CN-2h and pure $\mathrm{NH}_{4} \mathrm{Cl}$.

The XRD pattern of by-product shows that $\mathrm{NH}_{4} \mathrm{Cl}$ was present in the by-product. Otherwise, the broad peak range from $25^{\circ}$ to $28^{\circ}$ is consistent with main peaks of Cs-CN-2h, indicating there are some small fragment in the by-product from Cs-CN-2h. 


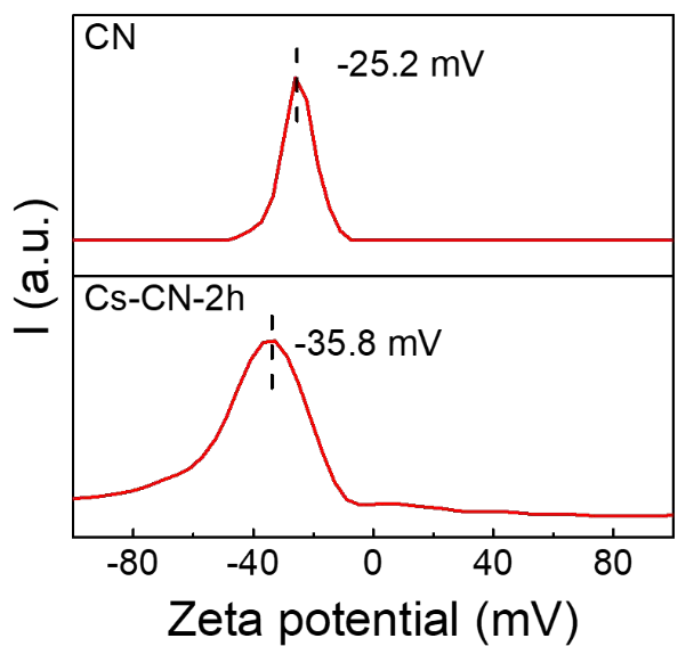

Figure S4 Zeta potential of CN and Cs-CN-2h.

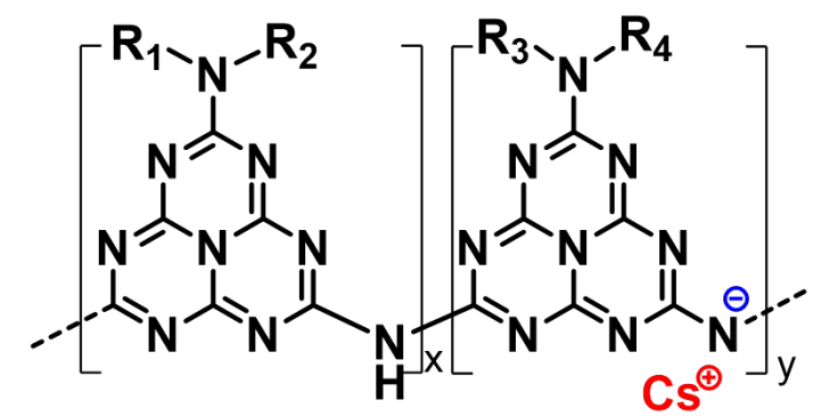

Figure S5. The suggested position of Cs ion in Cs-CN-2h 


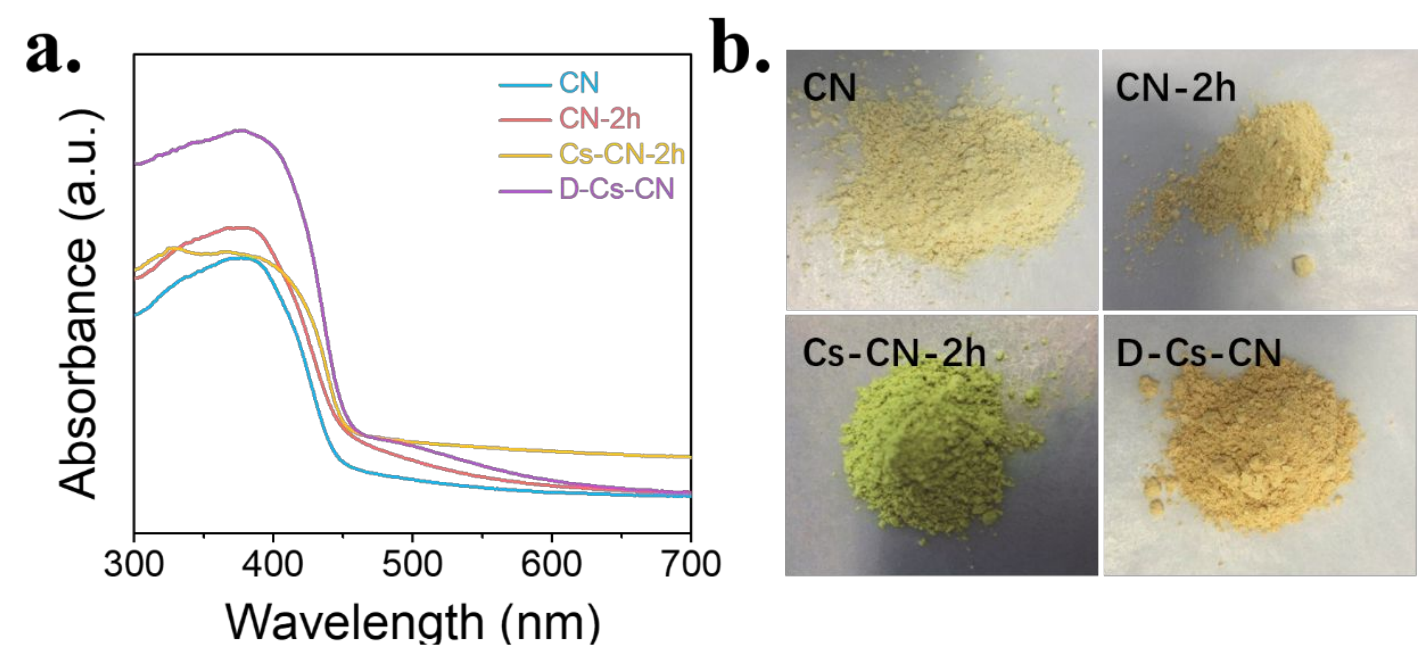

Figure S6. (a) Diffuse reflectance spectra of samples. (b) The photographs of samples.
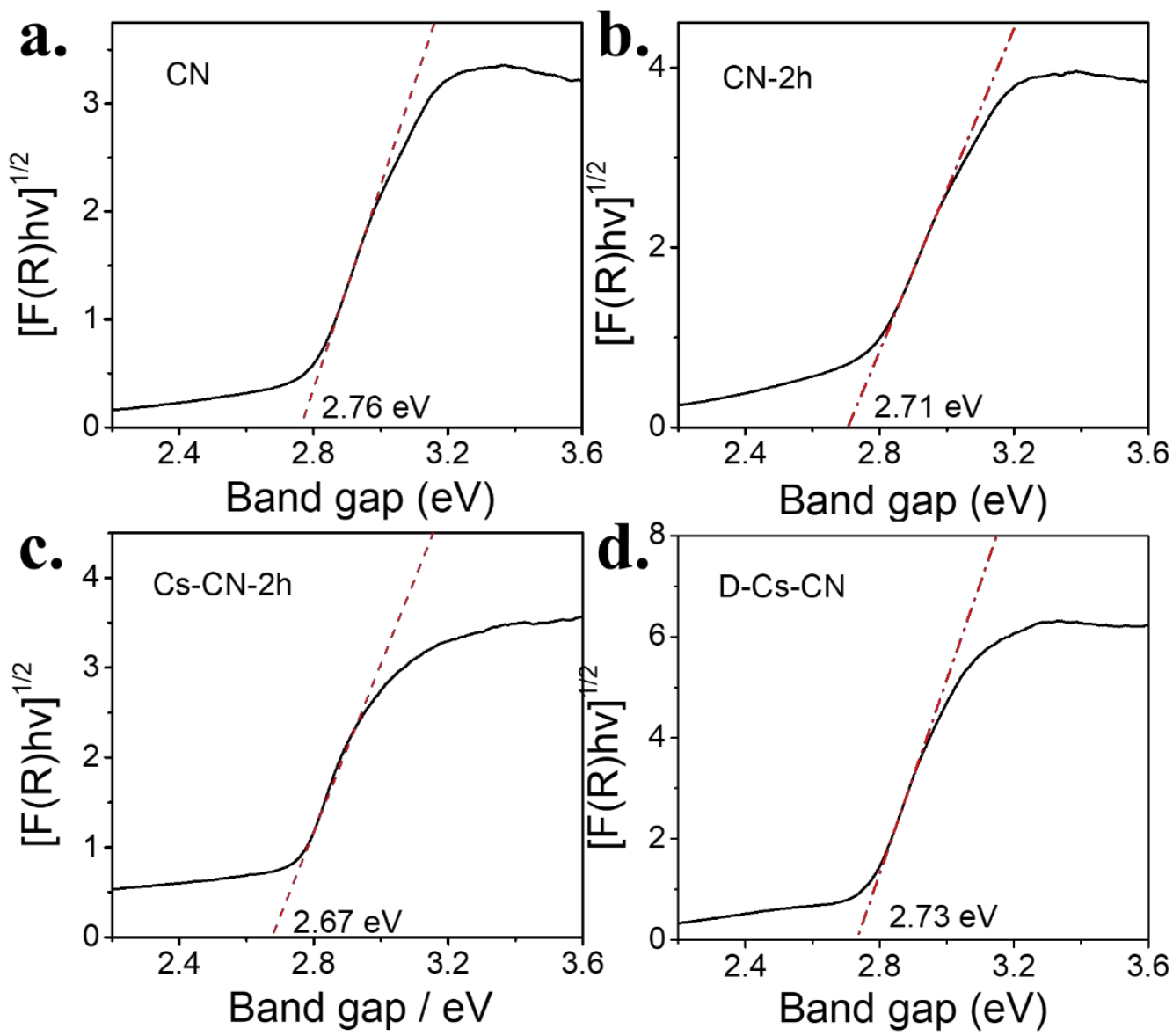

Figure S7. Kubelka-Munk plots of the samples derived from UV-Vis diffuse reflectance spectra in Figure S6. 

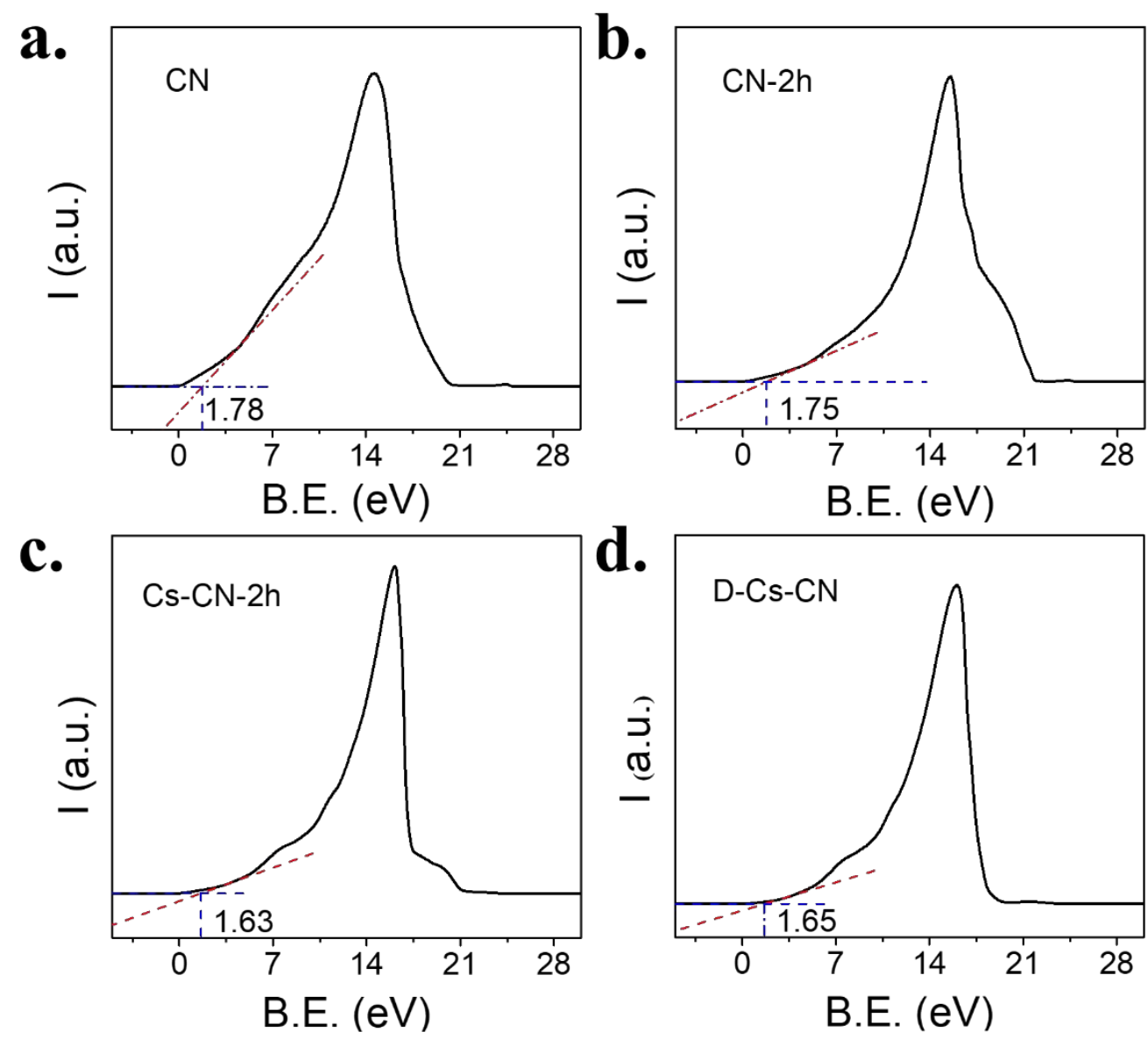

Figure S8. Valence band (VB) of the samples calculated from Ultraviolet Photoelectron Spectrometer (UPS).

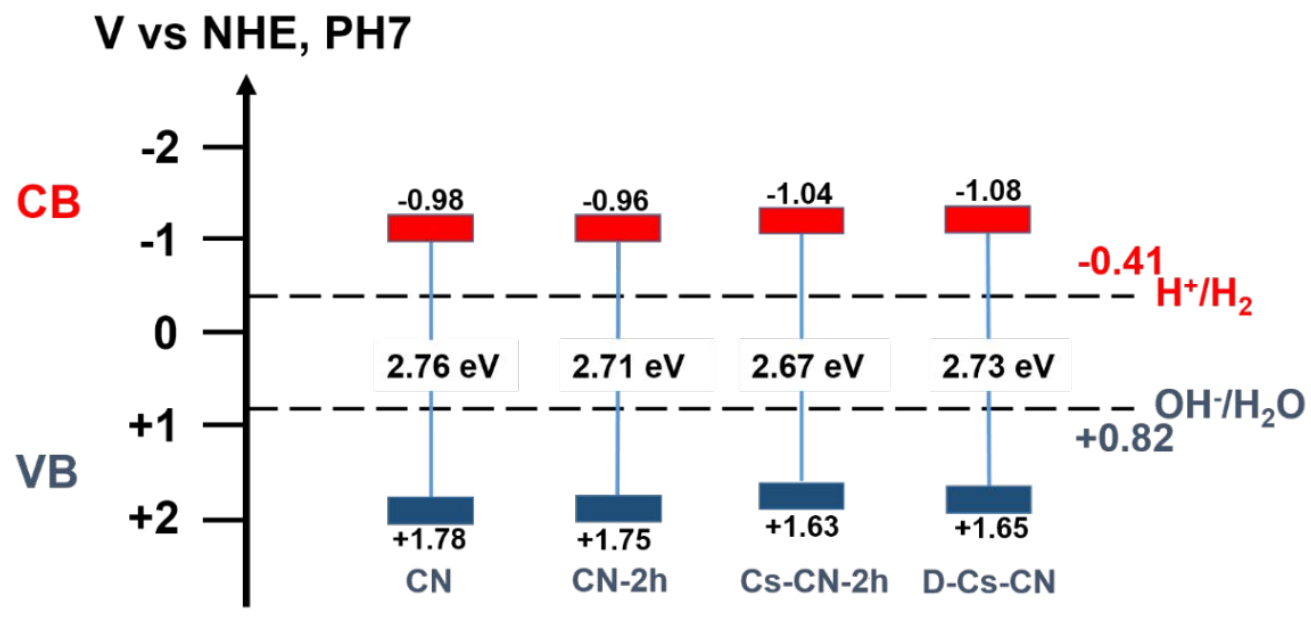

Figure S9. The electronic band structure of samples derived from Figure S7 and Figure S8. 


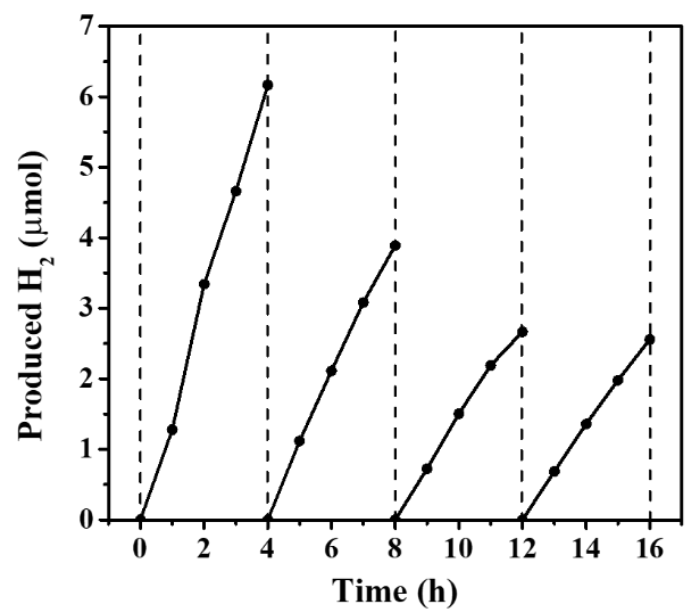

Figure S10. The cycle stability test of Cs-CN-2h.

The hydrogen production declined in the first three cycles and then stabilized in the fourth cycle. According to Table S5, The falling performance in first three cycles may due to two reasons. One is shedding of platinum because of vigorous stirring conditions, the other reason is $\mathrm{Cs}^{+}$ion can be exchanged by other positive ions because of its exchange properties of poly(heptazine imides). ${ }^{1}$ Furthermore, despite the decrease in stability, hydrogen production of Cs-CN-2h is still ten times higher than that of $\mathrm{CN}$.

Table S5. The elemental composition from XPS.

\begin{tabular}{ccccccc}
\hline Samples & C atm.\% & N atm.\% & O atm.\% & Cs atm.\% & Cl atm.\% & Pt atm.\% \\
\hline Cs-CN-2h & 42.29 & 48.33 & 4.12 & 4.80 & 0.27 & \\
$\mathrm{R}_{0 \mathrm{~h}}$-Cs-CN-2h & 67.14 & 17.62 & 13.06 & 1.05 & 0.08 & 1.03 \\
$\mathrm{R}_{4 \mathrm{~h}}$-Cs-CN-2h & 71.17 & 14.20 & 12.99 & 0.72 & 0.08 & 0.82 \\
\hline
\end{tabular}

Notes: $\mathrm{R}_{0 \mathrm{~h}}-\mathrm{Cs}-\mathrm{CN}-2 \mathrm{~h}$ means $\mathrm{Cs}-\mathrm{CN}-2 \mathrm{~h}$ irradiated for one hour to load it with platinum, and $\mathrm{R}_{4 h}-\mathrm{Cs}-\mathrm{CN}-2 \mathrm{~h}$ means $\mathrm{R}_{0 \mathrm{~h}}-\mathrm{Cs}-\mathrm{CN}-2 \mathrm{~h}$ reacted for four hours. 


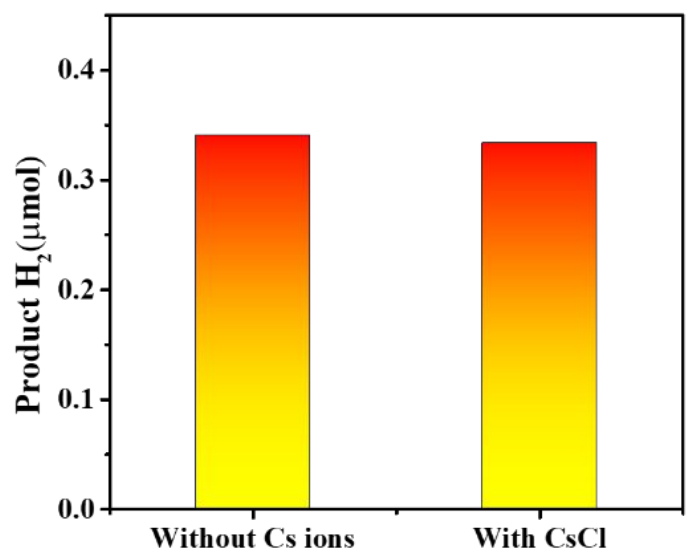

Figure $\mathrm{S} 11 . \mathrm{H}_{2}$ production comparison in first 4 hours between $\mathrm{CN}$ and $\mathrm{CN}$ with $\mathrm{CsCl}(5 \mathrm{wt} . \%$ Cs of $\mathrm{CN}$, just like the Cs content of Cs-CN-2h) under visible light ( $>420 \mathrm{~nm})$ irradiation, using TEOA as sacrificial agent and $2 \mathrm{wt} . \%$ of Pt as cocatalyst.

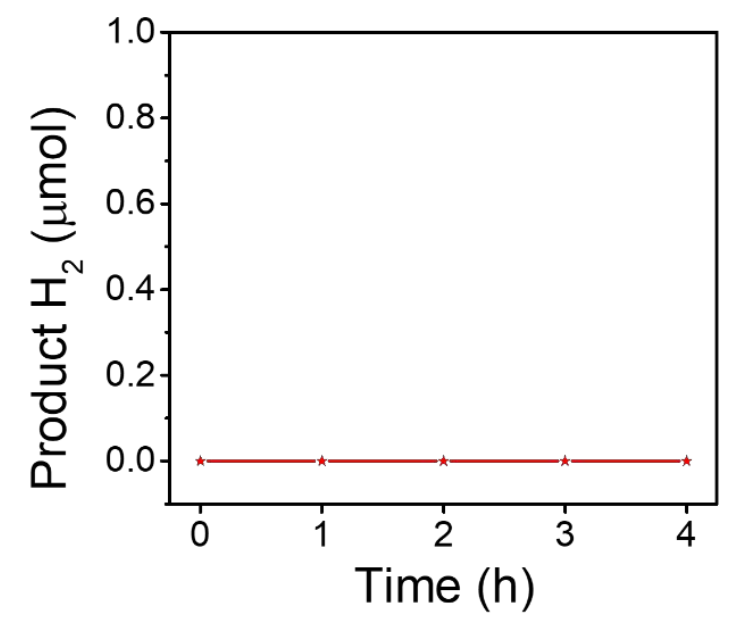

Figure S12. The product $\mathrm{H}_{2}$ for Cs-CN-2h in first four hours under dark condition using 4 mg samples, triethanolamine $(10 \mathrm{v} \%)$ as sacrificial agent, and $\mathrm{Pt}(2 \mathrm{wt} . \%)$ as cocatalyst. 


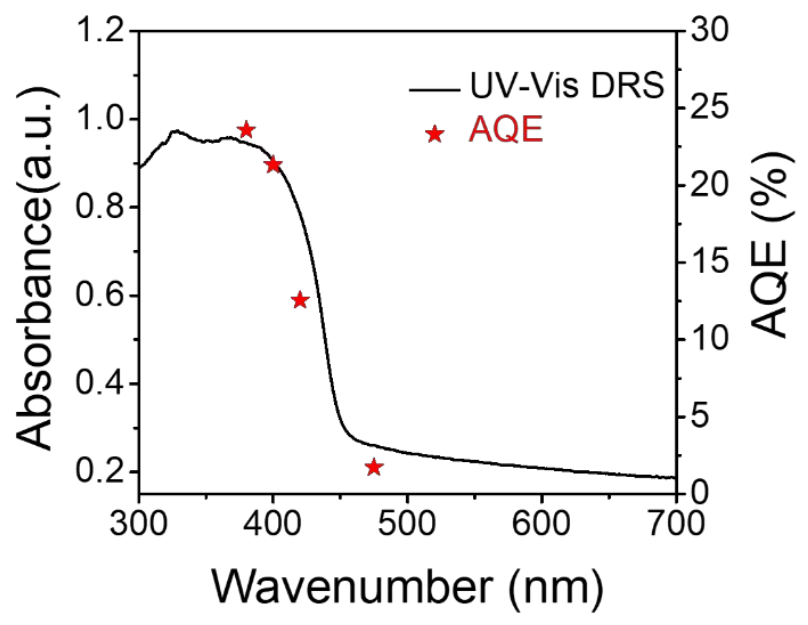

Figure S13 Wavelength dependent apparent quantum efficiency (AQE) of $\mathrm{H}_{2}$ evolution over Cs-CN-2h (right axis), UV/vis light absorption spectra of Cs-CN-2h (left axis).

Figure S13 shows the wavelength dependence of the AQE values of Cs-CN-2h for $\mathrm{H}_{2}$ evolution, thus indicating the correspondence of its variation tendencies of AQE curves with its UV-Vis DRS. The AQE value at $420 \mathrm{~nm}$ is estimated to be about $12.56 \%$. These results prove that the $\mathrm{H}_{2}$ evolution rate of the Cs-CN-2h was strongly dependent on the wavelength of the incident light. 

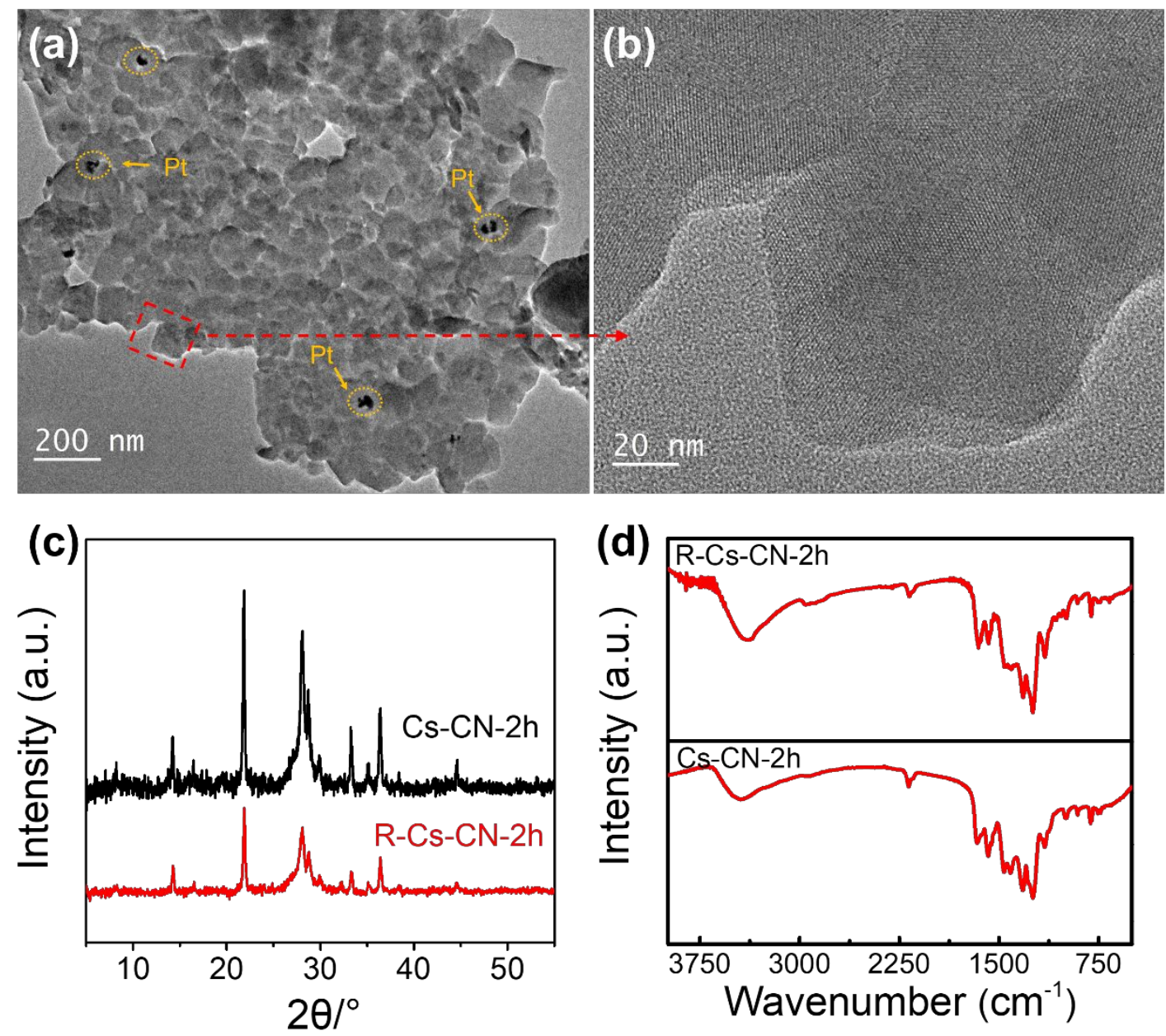

Figure S14 (a), (b) The TEM of Cs-CN-2h after reaction with 4h (named R-Cs-CN-2h). The XRD (c) and FT-IR (d) of Cs-CN-2h before and after the reaction.

As shown in Figure S14, there is little change for the morphology and structure of Cs-CN$2 \mathrm{~h}$ before and after the reaction.

\section{REFERENCES}

(1) Savateev, A.; Pronkin, S.; Willinger, M. G.; Antonietti, M.; Dontsova, D., Towards organic zeolites and inclusion catalysts: heptazine imide salts can exchange metal cations in the solid state. Chem. Asian J. 2017, 12, 1517-1522, DOI 10.1002/asia.201700209. 